\title{
Cytarabine-Daunorubicin-Etoposide Regimen
}

National Cancer Institute

\section{Source}

National Cancer Institute. Cytarabine-Daunorubicin-Etoposide Regimen. NCI Thesaurus.

Code C63613.

A regimen consisting of cytarabine, daunorubicn, and etoposide used as an induction regimen for the treatment of acute myeloid leukemia. 